\title{
Dressed perturbation theory: perturbative approach to Dyson-Schwinger and Bethe-Salpeter equations
}

\author{
Gastão Krein* \\ Instituto de Física Teórica, Universidade Estadual Paulista \\ Rua Dr. Bento Teobaldo Ferraz, 271 - Bloco II, 01140-070 São Paulo, SP - Brazil \\ E-mail: gkrein@ift.unesp.br
}

\begin{abstract}
Dressed perturbation theory (DPT) is a perturbative approach based on the idea of using dressed propagators, instead of free propagators like in ordinary perturbation theory. Quark and gluon self-energies are added to the free part of the QCD action and subtracted from the interaction part. The added self-energies are determined self-consistently via a devised symmetry-preserving power counting scheme. Correlation functions are then calculated using the modified QCD interaction according to the devised power counting. At zeroth order, DPT is very similar to the rainbow-ladder truncation scheme for Dyson-Schwinger and Bethe-Salpeter equations; the first nontrivial perturbative contribution introduces vertex corrections. Dynamical chiral symmetry breaking is realized in the light-quark sector; Goldstone's theorem and the axial-vector WardTakahashi identity are satisfied in the chiral limit. Higher order contributions can be calculated in a systematic, symmetry-preserving and controllable way, like in ordinary perturbation theory.
\end{abstract}

QCD-TNT-III-From quarks and gluons to hadronic matter: A bridge too far?,

2-6 September, 2013

European Centre for Theoretical Studies in Nuclear Physics and Related Areas (ECT*), Villazzano, Trento (Italy)

\footnotetext{
*Speaker.
} 


\section{Introduction and Motivation}

Dyson-Schwinger equations (DSEs) provide an ab initio framework to study in continuum space-time strongly interacting phenomena governed by Quantum Chromodynamics (QCD). They form an infinite hierarchy of coupled integral equations for correlation functions of quark and gluon fields. Bound states of quarks and gluons are identified as poles in the correlation functions; the amplitudes describing projections onto Fock-space components of the constituents are described by Bethe-Salperter equations (BSE). Because of the infinite hierarchy of the equations, truncation schemes must be devised to obtain a tractable problem. Ref. [1] surveys contemporary studies within such a framework, addressing progresses achieved with well known truncation schemes and identifying instances where they fail. For an earlier review, see Ref. [2].

In the present communication we present a novel truncation scheme for the hierarchy of DSEs in QCD based on perturbation theory; we name it Dressed Perturbation Theory (DPT). In ordinary perturbation theory, correlation functions are calculated as a power series in the QCD coupling constant, using noninterating quark and gluon propagators. In DPT, one uses dressed quark and gluon propagators that incorporate interactions via quark and gluon self-energies. The self-energiesare added to the original noninteracting part of the QCD Lagrangian, while the perturbative expansion is implemented with the original QCD interaction modified by the subtraction of the same quark and gluon self-energies that were added to the noninteracting part of the Lagrangian. The added quark and gluon self-energies are determined self-consistently via a devised symmetry-preserving power counting scheme. At zeroth order, DPT is very similar to the rainbow-ladder truncation scheme, in that quark and gluon self-energies are determined self-consistently via a DSE with bare quark-gluon vertices [1,2]. The first perturbative contribution in DPT introduces vertex corrections, similar to those studied in Refs. [3, 4, 5, 6, 7, 8, 9, 10, 11]. Higher order corrections can be calculated in systematic and controllable way, very much similar to ordinary perturbation theory.

The general idea behind DPT is not new. It shares many similarities with the method of the $\delta$-expansion, also known as optimised perturbation theory, used with self-interacting scalar field theories [12], relativistic nuclear mean-field theories [13], and dynamical phase transitions in the context of Ginzburg-Landau-Langevin equations [14]. It also shares similarities with screened perturbation theory [15], that has been used in finite temperature field theory. In these, the selfenergies added to the noninteracting part of the action and subtracted from the original interacting part are constant mass terms; in DPT they are momentum-dependent, an essential feature for the construction of symmetry-preserving BSE kernels. As we show in the present communication, this is a key feature of DPT, as it describes dynamical chiral symmetry breaking (D $\chi \mathrm{SB})$ in the light-quark sector, in that the the pion is obtained as the Goldstone boson in the chiral limit, and the axial-vector Ward-Takahashi identity is preserved.

The long-term goals of DPT are applications in heavy-quark sector of QCD, with special interest in heavy-light systems, like $D$ and $B$ mesons. It is a known feature of the rainbow-ladder scheme that fails to reproduce the experimental values of the electroweak decay constants of $D$ and $B$ mesons, despite describing well their masses. The discrepancies are of the order of $25 \%$, as discussed in the review presented in Ref. [16]. The authors speculated that a possible reason for this failure could be that the rainbow-ladder truncation is not reliable in the charm quark region. The issue is of immense contemporary interest for ongoing heavy-ion experiments and forthcoming 
experiments to be conducted at FAIR and JLab. In particular, there is interest in low-energy $D N$ cross sections [17], and of exotic nuclear bound states of $J / \Psi$ [18]. In such studies, there is particular interest is the question of flavor symmetry breaking in hadron couplings [19].

\section{DPT for Dyson-Schwinger Equations}

Let us start from the QCD action $\mathscr{S}=\mathscr{S}_{0}+\mathscr{S}_{1}$, where $\mathscr{S}_{0}$ is quadratic in the fields

$$
\mathscr{S}_{0}=\int d^{4} x d^{4} y \bar{\psi}(x) S_{0}^{-1}(x-y) \psi(y)+\frac{1}{2} \int d^{4} x d^{4} y A_{\mu}^{a}(x)\left(D_{0}^{-1}\right)_{\mu v}^{a b}(x-y) A_{v}^{b}(y),
$$

and $\mathscr{S}_{I}$ contains the usual quark-gluon and three- and four-gluon interactions ${ }^{1}$ :

$$
\begin{aligned}
\mathscr{S}_{I}= & \int d^{4} x\left[i g \bar{\psi}(x) \gamma \cdot A^{a} T^{a} \psi(x)-g f^{a b c} \partial_{\mu} A_{v}^{a}(x) A_{\mu}^{b}(x) A_{v}^{c}(x)\right. \\
& \left.+\frac{1}{4} g^{2} f^{a b c} f^{a d e} A_{\mu}^{b}(x) A_{v}^{c} A_{\mu}^{d}(x) A_{v}^{e}(x)\right],
\end{aligned}
$$

where

$$
\begin{aligned}
S_{0}^{-1}(x-y) & =\left(\gamma_{\mu} \partial_{\mu}+m\right) \delta^{(4)}(x-y), \\
\left(D_{0}^{-1}\right)_{\mu \nu}^{a b}(x-y) & =\delta^{a b}\left[-\delta_{\mu \nu} \partial^{2}+\left(1-\frac{1}{\xi}\right) \partial_{\mu} \partial_{\nu}\right] \delta^{4}(x-y),
\end{aligned}
$$

where $\xi$ is a covariant-gauge fixing parameter.

Ordinary perturbation theory for correlation functions, like the quark and gluon propagators, is based on a power series expansion in the coupling constant $g$. The correlation functions calculated in this way are expressed in terms of the zeroth-order propagators $S_{0}$ and $D_{0}$, whose inverses are given in Eqs. (2.4) and (2.5). The basic main idea of DPT is to use dressed propagators, instead of the zeroth order ones, and the series expansion is made with a modified interaction, not the original $\mathscr{S}_{I}$. Specifically, DPT consists in three main steps:

- Quark and gluon self-energies, quadratic in the quark and gluon fields, are added to $\mathscr{S}_{0}$ and subtracted from $\mathscr{S}_{I}$ :

$$
\begin{gathered}
\mathscr{S}_{0} \rightarrow \overline{\mathscr{S}}_{0}=\mathscr{S}_{0}+\langle\bar{\psi} \bar{\Sigma} \psi\rangle+\left\langle A_{\mu} \bar{\Pi}_{\mu v} A_{v}\right\rangle, \\
\mathscr{S}_{I} \rightarrow \overline{\mathscr{S}}_{I}=\mathscr{S}_{I}-\langle\bar{\psi} \bar{\Sigma} \psi\rangle-\left\langle A_{\mu} \bar{\Pi}_{\mu v} A_{v}\right\rangle,
\end{gathered}
$$

where $\langle\bar{\psi} \bar{\Sigma} \psi\rangle$ and $\langle A \bar{\Pi} A\rangle$ are shorthand notations for double integrals involving the quark and gluon fields and self-energies that will be spelled out shortly ahead. This will not change the original action, as $\overline{\mathscr{S}}_{0}+\overline{\mathscr{S}}_{I}=\mathscr{S}_{0}+\mathscr{S}_{I}=\mathscr{S}$;

\footnotetext{
${ }^{1}$ The SU(N) generators are $T^{a}=\lambda^{a} / 2, a=1, \cdots, N^{2}-1$, with normalization $\operatorname{Tr}\left(T^{a} T^{b}\right)=\delta^{a b} / 2$, and $\left[T^{a}, T^{b}\right]=$ ${ }_{i f}^{a b c} T^{c},\left(T^{a}\right)^{i k}\left(T^{a}\right)^{k j}=C_{F} \delta^{i j}, f^{i k l} f^{j k l}=C_{A} \delta^{i j}, C_{F}=\left(N^{2}-1\right) / 2 N, C_{A}=N$. Euclidean metric conventions are: $A \cdot B=\sum_{i=1}^{4} A_{i} B_{i} ;\left\{\gamma_{\mu}, \gamma_{v}\right\}=2 \delta_{\gamma_{v}} ; \gamma_{\mu}^{\dagger}=\gamma_{\mu} ; \gamma_{5}=-\gamma_{1} \gamma_{2} \gamma_{3} \gamma_{4}$ :

$$
\gamma_{1,2,3}=\left(\begin{array}{cc}
0 & -i \sigma^{1,2,3} \\
i \sigma^{1,2,3} & 0
\end{array}\right), \gamma_{4}=\left(\begin{array}{cc}
1 & 0 \\
0 & -1
\end{array}\right) \text {, }
$$

where $\sigma^{1}, \sigma^{2}, \sigma^{3}$ are the Pauli matrices. Einstein's convention of summation over repeated indices is used in the paper.
} 
- Correlation functions are calculated as a power series in $\overline{\mathscr{S}}_{I}$;

- A criterion is defined to fix self-consistently the self-energies $\Sigma$ and $\Pi$.

The first step leads to the following expressions for $\overline{\mathscr{S}}_{0}$ and $\overline{\mathscr{S}}_{I}$ :

$$
\overline{\mathscr{S}}_{0}=\int d^{4} x d^{4} y \bar{\psi}(x) \bar{S}^{-1}(x-y) \psi(y)+\frac{1}{2} \int d^{4} x d^{4} y A_{\mu}^{a}(x)\left(\bar{D}^{-1}\right)_{\mu v}^{a b}(x-y) A_{v}^{b}(y),
$$

with

$$
\begin{gathered}
\bar{S}^{-1}(x-y)=S_{0}^{-1}(x-y)+\bar{\Sigma}(x-y), \\
\left(\bar{D}^{-1}\right)_{\mu v}^{a b}(x-y)=\left(D_{0}^{-1}\right)_{\mu v}^{a b}(x-y)+\bar{\Pi}_{\mu v}^{a b}(x-y),
\end{gathered}
$$

and

$$
\begin{aligned}
\overline{\mathscr{S}}_{I}= & \delta \int d^{4} x\left[i g \bar{\psi}(x) \gamma \cdot A^{a} T^{a} \psi(x)-g f^{a b c} \partial_{\mu} A_{v}^{a}(x) A_{\mu}^{b}(x) A_{v}^{c}(x)\right. \\
& \left.+\frac{1}{4} \delta g^{2} f^{a b c} f^{a d e} A_{\mu}^{b}(x) A_{v}^{c} A_{\mu}^{d}(x) A_{v}^{e}(x)\right] \\
& -\delta^{2} \int d^{4} x d^{4} y\left[\bar{\psi}(x) \bar{\Sigma}(x-y) \psi(y)-\frac{1}{2} A_{\mu}^{a}(x) \bar{\Pi}_{\mu v}^{a b}(x-y) A_{v}^{b}(y)\right] .
\end{aligned}
$$

The parameter $\delta$ here is introduced for bookkeeping purposes in the implementation of the second step of DPT: correlation functions and observables are calculated as a power series in $\delta$ and, at the end of the calculation, $\delta$ is put equal to one. It is important to note that an expansion in $\delta$ is not equivalent to an expansion in $g$, as the added self-energies $\bar{\Sigma}$ and $\bar{\Pi}_{0 \mu \nu}$ will turn out to be non analytic functions of $g$. Also, $\delta$ is not meant to be a "small expansion parameter"; as said, it is introduced to control the expansion. It is $\overline{\mathscr{S}}_{I}$ that is meant to be "small", in the sense that contributions to correlation functions and observables calculated perturbatively with $\overline{\mathscr{S}}_{I}$ will become smaller and smaller as the order in $\delta$ is increased.

The third step of DPT consists in defining a criterion for fixing the self energies $\Sigma_{0}$ and $\Pi_{0 \mu \nu}$. One possible criterion is the following. Quark and gluon self-energies calculated perturbatively with $\overline{\mathscr{S}}_{I}$ will be given as a power series in $\delta$ :

$$
\begin{aligned}
\Sigma & =\bar{\Sigma}+\delta^{2} \Sigma_{2}+\delta^{4} \Sigma_{4}+\cdots \\
\Pi_{\mu v} & =\bar{\Pi}_{\mu v}+\delta^{2} \Pi_{2 \mu v}+\delta^{4} \Pi_{4 \mu v}+\cdots
\end{aligned}
$$

where the zeroth order self energies $\bar{\Sigma}$ and $\bar{\Pi}_{\mu \nu}$ are those introduced in Eqs. (2.6) and (2.7). Note that from the $\delta$ dependence of $\overline{\mathscr{S}}_{I}$, there are no odd powers of $\delta$ contributing to the quark and gluon self energies. As in ordinary perturbation theory, calculations are most easily done in momentum space. The zeroth order propagators in momentum space of DPT are given in terms of the Fourier 
transforms $\bar{\Sigma}(p)$ and $\bar{\Pi}_{\mu v}(p)$ of respectively $\bar{\Sigma}(x-y)$ and $\bar{\Pi}_{\mu v}(x-y)$ by:

$$
\begin{aligned}
\bar{S}(p) & =\frac{1}{i \gamma \cdot p+m+\bar{\Sigma}(p)} \\
\bar{D}_{\mu v}(p) & =\left(\delta_{\mu v}-\frac{p_{\mu} p_{v}}{p^{2}}\right) \frac{1}{p^{2}\left[1+\bar{\Pi}\left(p^{2}\right)\right]}+\xi \frac{p_{\mu} p_{v}}{p^{4}},
\end{aligned}
$$

where $\bar{\Pi}$ is defined via $\bar{\Pi}_{\mu v}\left(p^{2}\right)=\left(\delta_{\mu v} p^{2}-p_{\mu} p_{v}\right) \bar{\Pi}\left(p^{2}\right)$. The $\bar{\Sigma}$ and $\bar{\Pi}_{\mu v}$ can be fixed by imposing that the first non trivial contributions to $\Sigma(p)$ and $\Pi_{\mu v}$ in Eqs. (2.12) and (2.13) vanish, that is:

$$
\Sigma_{2}(p)=0 \quad \text { and } \quad \Pi_{2 \mu v}=0 .
$$

The second order contributions $\Sigma_{2}(p)$ and $\Pi_{2 \mu v}$ are depicted in Fig. 1.

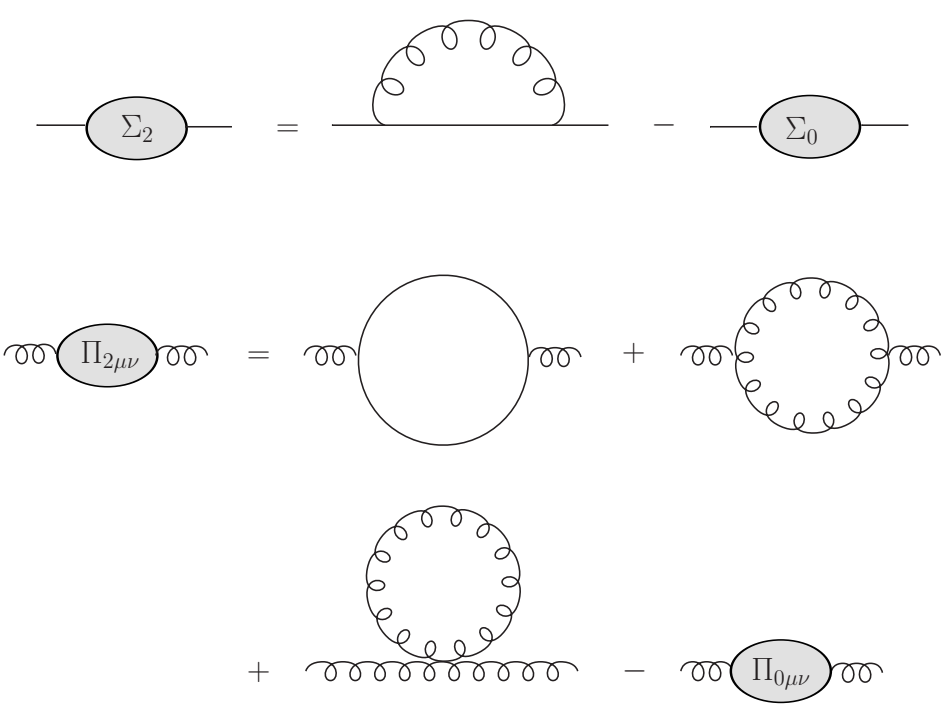

Figure 1: Diagrammatic representation of $\Sigma_{2}$ (upper graphs) and $\Pi_{2 \mu \nu}(p)$ (lower graphs). The solid and curly lines represent the dressed propagators $\bar{S}$ and $\bar{D}$, respectively.

The imposed conditions $\Sigma_{2}=0$ and $\Pi_{2 \mu v}=0$ lead to the following self-consistent coupled integral equations for $\Sigma_{0}(p)$ and $\Pi_{0 \mu \nu}(p)$ (for two flavors):

$$
\begin{aligned}
& \bar{\Sigma}(p)=\frac{4}{3} g^{2} \int \frac{d^{4} q}{(2 \pi)^{4}} \bar{D}_{\mu v}(p-q) \gamma_{\mu} \bar{S}(q) \gamma_{v} \\
& \bar{\Pi}_{\mu v}(p)=-g^{2} \int \frac{d^{4} q}{(2 \pi)^{4}} \operatorname{Tr}_{D}\left[\gamma_{\mu} \bar{S}(p+q) \gamma_{v} \bar{S}(q)\right]+\operatorname{CONST}_{\text {tad }} \\
& +\frac{3}{2} g^{2} \int \frac{d^{4} q}{(2 \pi)^{4}} N_{\mu \rho \sigma}(p,-p-q, q) \bar{D}_{\sigma \sigma^{\prime}}(q) \bar{D}_{\rho \rho^{\prime}}(p+q) N_{\sigma^{\prime} \rho^{\prime} v}(q,-(p+q), p),
\end{aligned}
$$

where $\operatorname{Tr}_{D}$ means trace over Dirac spinor indices, $\mathrm{CONST}_{\text {tad }}$ is a constant from the four-gluon tadpole and $N_{\mu v \rho}$ is the bare three-gluon vertex (all gluon momenta entering the vertex),

$$
N_{\mu v \rho}(k, p, q)=-i(k-p)_{\rho} \delta_{\mu v}-i(p-q)_{\mu} \delta_{v \rho}-i(q-k)_{\nu} \delta_{\mu \rho} .
$$


Fig. 2 is a graphical representation these two equations. They are self-consistent because the unknowns $\Sigma_{0}$ and $\Pi_{0 \mu \nu}$ are contained in the zeroth-order propagators $\bar{S}$ and $\bar{D}_{\mu \nu}$ in the integrals.

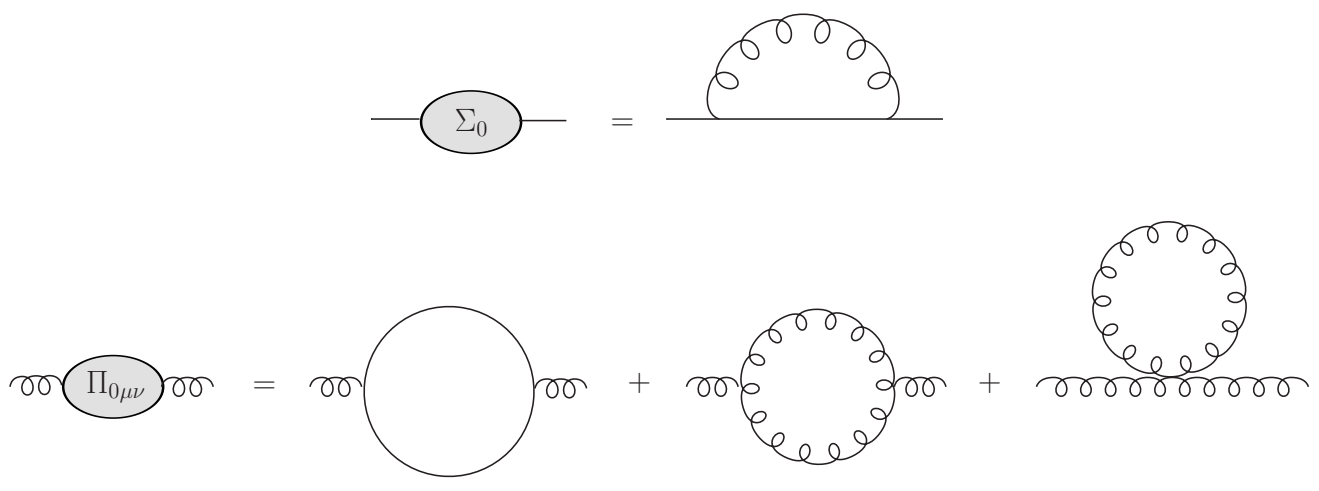

Figure 2: Diagrammatic representation of the dressed rainbow and random phase approximations for the quark (upper graphs) and the gluon self-energies (lower graphs).

Eq. (2.17) - depicted by the upper graph in Fig. 2 - is the dressed rainbow approximation for the quark propagator; the difference from the usual rainbow approximation is that $\bar{D}_{\mu \nu}^{(0)}$ is not the bare gluon propagator. Similarly, Eq. (2.19) - lower graph in Fig. 2 - gives the dressed random phase approximation for the gluon propagator; a generalized random phase approximation, in that quark and gluon bubbles are summed with self-consistent dressed quark and gluon propagators.

Eqs. (2.17) and (2.19) provide a starting point for DPT, as the zeroth order has been defined and higher order contributions can be calculated systematically like in ordinary perturbation theory. The next order of contribution to the quark and gluon self energies are $\mathscr{O}\left(\delta^{4}\right)$. At this order, the (internal) quark and gluon lines in Fig. 2 get dressed with insertions of the $\mathscr{O}\left(\delta^{2}\right)$ self-energies $\Sigma_{2}$ and $\Pi_{2 \mu v}$, and new graphs representing dressing of the quark-gluon and three- and four-gluon gluon vertices appear. Because of lack of space, from now on we will concentrate on the quark propagator.

The $\mathscr{O}\left(\delta^{4}\right)$ contributions to the quark self-energy are are depicted in Fig. 3. When imposing Eqs. (2.17) and (2.19) on the inserted $\mathscr{O}\left(\delta^{2}\right)$ self-energies, the two first graphs vanish and only the quark-gluon vertex corrections (two last graphs) contribute to $\Sigma_{4}$. The same feature happens with the $\mathscr{O}\left(\delta^{4}\right)$ contributions to the gluon self-energy, the $\mathscr{O}\left(\delta^{2}\right)$ on internal quark and gluon lines vanish and only vertex corrections contribute. One of them is similar to the (Abelian) one-photon vertex correction in QED, which we denote $\Sigma_{4}^{A}$, and the other contribution is a three-gluon vertex (non-Abelian), which we denote $\Sigma_{4}^{n A}$; they are given by

$$
\Sigma_{4}^{A}(p)=\frac{N_{c}^{2}-1}{4 N_{c}^{2}} g^{4} \int \frac{d^{4} q}{(2 \pi)^{4}} \int \frac{d^{4} l}{(2 \pi)^{4}} \bar{D}_{\mu v}(p-q) \gamma_{\mu} \bar{S}(q) \gamma_{\rho} \bar{S}(q+l) \gamma_{v} \bar{S}(p+l) \bar{D}_{\rho \sigma}(l) \gamma_{\sigma},
$$

and

$$
\begin{aligned}
\Sigma_{4}^{n A}(p)= & -i \frac{N_{c}^{2}-1}{4} g^{4} \int \frac{d^{4} q}{(2 \pi)^{4}} \int \frac{d^{4} l}{(2 \pi)^{4}} \bar{D}_{\mu v}(p-q) \gamma_{\mu} \bar{S}(q) N_{v \rho \sigma}(p-q, q-l, l-p) \\
& \times \gamma_{\sigma^{\prime}} \bar{D}_{\sigma^{\prime} \rho}(q-l) \bar{S}(l) \gamma_{\rho^{\prime}} \bar{D}_{\sigma^{\prime} \sigma}(l-p) .
\end{aligned}
$$


Here we have written explicitly the dependence on the number of colors $N_{c}$; as can be seen, the Abelian contribution is $1 / N_{c}^{2}$ suppressed with respect to the non-Abelian contribution. A simple application of DPT to a contact gluon propagator has been made in Ref. [20]. The dominance of the non-Abelian vertex over over the Abelian correction can be nicely seen.

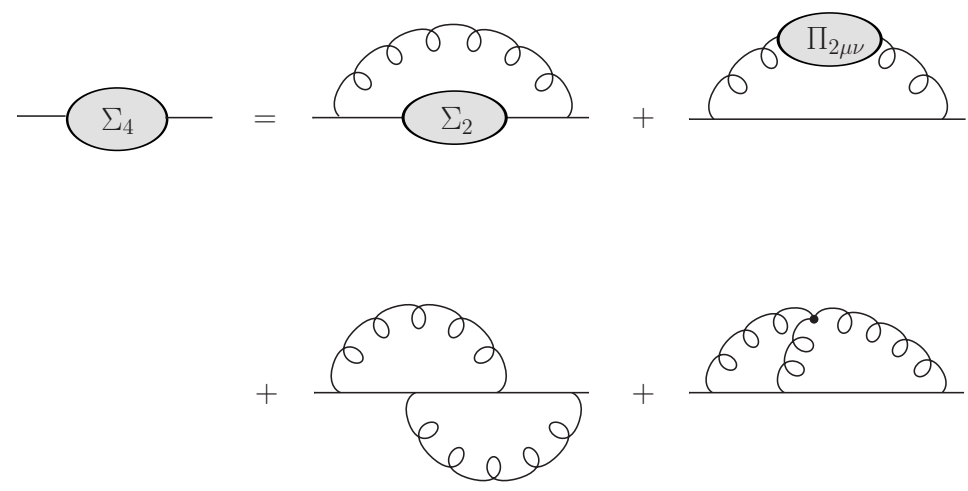

Figure 3: Diagrammatic representation of the $\mathscr{O}\left(\delta^{4}\right)$ to the quark self-energy. The solid and curly lines represent the dressed propagators $\bar{S}$ and $\bar{D}$, respectively.

From the exact DSE for the quark self-energy

$$
\Sigma(p)=\int \frac{d^{4} q}{(2 \pi)^{4}} g^{2} D_{\mu v}(p-q) \gamma_{\mu} T^{a} S(q) \Gamma_{v}^{a}(q, p),
$$

and Eqs. (2.21) and (2.22), one deduces the $\mathscr{O}\left(\delta^{4}\right)$ Abelian $\Gamma_{\mu}^{a A}$ and non-Abelian $\Gamma_{\mu}^{a n A}$ contributions to the full quark-gluon vertex :

$$
\Gamma_{\mu}^{a}=\gamma_{\mu} T^{a}+\Gamma_{\mu}^{a A}+\Gamma_{\mu}^{a n A}
$$

with

$$
\Gamma_{\mu}^{a A}(q, p)=-g^{2} T^{b} T^{a} T^{b} \int \frac{d^{4} l}{(2 \pi)^{4}} \gamma_{\rho} \bar{S}(q+l) \gamma_{\mu} \bar{S}(p+l) \bar{D}_{\rho \sigma}(l) \gamma_{\sigma}
$$

and

$$
\Gamma_{\mu}^{a n A}(q, p)=-g^{2} f^{a b c} T^{b} T^{c} \int \frac{d^{4} l}{(2 \pi)^{4}} N_{\mu \rho \sigma}(p-q, q-l, l-p) \gamma_{\sigma^{\prime}} \bar{D}_{\sigma^{\prime} \rho}(q-l) \bar{S}(l) \gamma_{\rho^{\prime}} \bar{D}_{\sigma^{\prime} \sigma}(l-p),
$$

where we made use of $T^{b} T^{a} T^{b}=-T^{a} / 2 N_{c}$ and $f^{a b c} T^{b} T^{c}=i T^{a} N_{c} / 2$. Here, one recognizes the (dressed) one-loop correction to the quark-gluon vertex.

Corrections $\mathscr{O}\left(\delta^{4}\right)$ to the gluon propagator are also vertex corrections. In order to maintain local gauge symmetry, explicit ghost fields must be considered. The same perturbation procedure can be implemented for ghosts; imposing that $\mathscr{O}\left(\delta^{2}\right)$ contributions vanish, like in Eq. (2.16) for the quark and gluon self-energies, the zeroth-order ghost self-energies become determined: they satisfy a self-consistent Dyson-Schwinger equation with bare vertices.

Like with other truncation schemes in QCD, an important issue that must be observed is the preservation of Ward identities related to global symmetries. Amongst all, the axial-vector WardTakahashi identity plays prominent role in connection with the approximate chiral symmetry of 
QCD in the light quark sector, and the identification of the pion as the associated pseudo NambuGoldstone boson as solution of the homogeneous Bethe-Salpeter in the isovector pseudoscalar channel. In the hypothetical chiral limit, the pion would be massless - which is the content of Goldstone's theorem. This the subject of the next section.

\section{Bether-Salpeter equations, Goldstone Theorem and Ward-Takahashi identities}

Meson bound states can be obtained from the homogeneous Bethe-Salpeter equation (BSE)

$$
\left[\Gamma_{M}(q ; P)\right]_{A B}=\int \frac{d^{4} k}{(2 \pi)^{4}}\left[K_{M}(q, k ; P)\right]_{A C, D B}\left[S\left(k_{+}\right) \Gamma_{M}(p, k ; P) S\left(k_{-}\right)\right]_{C D}
$$

where $K_{M}(q, k ; P)$ is the fully amputated quark-antiquark scattering kernel; $A, B, \cdots$ denote collectively color, flavor, and spinor indices; and $k_{ \pm}=k \pm \eta_{ \pm} P$, with $\eta_{+}+\eta_{-}=1$. Here, $k$ is relative momentum, and $P$ is total momentum; if $k_{1}$ and $k_{2}$ are the momenta of particles 1 and 2 , then $k_{1}=k+\eta_{+} P$, and $k_{2}=k-\eta_{-} P$. The mass of the meson is the eigenvalue $P^{2}=-M^{2}$. Let us consider the Bethe-Salpeter amplitude in the pion channel, $\Gamma_{M} \equiv \tau^{i} \Gamma_{\pi}^{i}$, where $\tau^{i}, i=1,2,3$, are the Pauli isospin matrices. The general structure (w.r.t. parity, charge conjugation, and Lorentz transformations) of $\Gamma_{\pi}$ is:

$$
\Gamma_{\pi}=\gamma_{5}\left(i E_{\pi}+\gamma \cdot P F_{\pi}+\gamma \cdot k \gamma \cdot P G_{\pi}+\sigma_{\mu \nu} q_{\mu} P_{v} H_{\pi}\right)
$$

where $E_{M}, F_{M}, \cdots$ are scalar functions of $q$ and $P$. In the chiral limit, $m=0$ in Eq. (2.4), D $\chi \mathrm{SB}$ demands that Eq. (3.1) has a solution with $P^{2}=0$ in the pseudoscalar channel.

Closely related to the pion bound state amplitude $\Gamma_{\pi}^{a}(q ; P)$, is the pseudovector vertex function $\Gamma_{5 \mu}^{a}(q ; P)$, which satisfies the inhomogeneous BSE (omitting a renormalization $Z_{2}$ factor multiplying the inhomogeneous term):

$$
\left[\Gamma_{5 \mu}^{i}(q ; P)\right]_{A B}=\left[\tau^{i} \gamma_{5} \gamma_{\mu}\right]_{A B}+\int \frac{d^{4} k}{(2 \pi)^{4}}\left[K_{M}(q, k ; P)\right]_{A C, D B}\left[S\left(k_{+}\right) \Gamma_{5 \mu}^{i}(k ; P) S\left(k_{-}\right)\right]_{C D} .
$$

The Ward-Takahashi identity for in the chiral limit $m=0$, reflecting the conservation of the axialvector current in QCD, is :

$$
P_{\mu} \Gamma_{5 \mu}^{i}(q ; P)=S^{-1}\left(q_{+}\right) i \gamma_{5} \tau^{i}+i \gamma_{5} \tau^{i} S^{-1}\left(q_{-}\right)
$$

Using here $S^{-1}(p)=i \gamma \cdot p+\Sigma(p)$, with $\Sigma(p)$ given by the exact expression in Eq. (2.23), one can rewrite the identity as

$$
\begin{aligned}
& \int \frac{d^{4} k}{(2 \pi)^{4}}\left\{g^{2} D_{\mu v}\left(q_{+}-k\right)\left[\gamma_{\mu} T^{a} S(k) \Gamma_{v}^{a}\left(k, q_{+}\right) \gamma_{5}\right]_{A B}+g^{2} D_{\mu v}\left(q_{-}-k\right)\left[\gamma_{5} \gamma_{\mu} T^{a} S(k) \Gamma_{v}^{a}\left(k, q_{-}\right)\right]_{A B}\right\} \\
& =\int \frac{d^{4} k}{(2 \pi)^{4}}[K(q, k ; P)]_{A C, D B}\left[S\left(k_{+}\right) \gamma_{5}+\gamma_{5} S\left(k_{-}\right)\right]_{C D} .
\end{aligned}
$$

This shows that there is a dynamical relation imposed by chiral symmetry between the quark selfenergy. 
The issue we address next is whether Goldstone's theorem and the Ward-Takahashi identity are obeyed order by order in DPT. When the exact DSE has a nonzero solution for the scalar part of the quark self-energy, then the exact BSE has a massless solution in the quark-antiquark pseudoscalar channel - the Nambu-Goldstone boson. A priori, since DPT provides an approximate treatment for the DSE, it is not obvious that a Nambu-Golsdstone boson will be obtained from the corresponding approximate DSE. The question can be paraphrased as follows: given the Dyson-Schwinger equation for the quark self-energy at zeroth order, what is the corresponding quark-antiquark scattering kernel that produces a Nambu-Gol dstone boson? The answer to this question is similar to the one provided in Ref. [3] in the context of an improved rainbow-ladder approximation: given a DSE for the quark self-energy, the kernel of the corresponding BSE equation is obtained via the replacement

$$
\gamma_{\mu} \bar{S}(k) \gamma_{v} \rightarrow \gamma_{\mu} \bar{S}\left(k_{+}\right) \Gamma_{\pi}(k ; P) \bar{S}\left(k_{-}\right) \gamma_{v} .
$$

That is, one cuts the internal dressed quark line in the quark self-energy graph to introduce the meson total momentum $P$. Let us examine this scheme in DPT at zeroth order.

We define, as usual

$$
S^{-1}(p)=i \gamma \cdot p+m+\Sigma(p) \equiv i \gamma \cdot p A\left(p^{2}\right)+B\left(p^{2}\right),
$$

where $A\left(p^{2}\right)$ and $B\left(p^{2}\right)$ are Lorentz scalar functions. In the chiral limit $(m=0), \mathrm{D} \chi \mathrm{SB}$ is characterised by a nonzero solution of the integral equation for the scalar part of the quark self-energy, i.e. $B\left(p^{2}\right) \neq 0$. At zeroth order, Eq. (2.17), the integral equation for $\bar{B}\left(p^{2}\right)$ is

$$
\bar{B}\left(p^{2}\right)=\frac{16}{3} g^{2} \int \frac{d^{4} q}{(2 \pi)^{4}} \bar{D}_{\mu \mu}(p-q) \frac{\bar{B}\left(q^{2}\right)}{q^{2} \bar{A}^{2}\left(q^{2}\right)+\bar{B}^{2}\left(q^{2}\right)} .
$$

At this level of approximation, the scheme of Ref. [3] leads to the following kernel for the BSE

$$
\left[K_{M}(q, k ; P)\right]_{A C, D B}=-g^{2}\left(\gamma_{\mu} T^{a}\right)_{A C} \bar{D}_{\mu v}(q-k)\left(\gamma_{v} T^{a}\right)_{D B} .
$$

Clearly, this amounts to cutting the internal dressed quark line in the quark self-energy graph in Fig. 2 - top graph. Replacing this kernel into Eq. (3.1) and putting $P=0$ in Eq. (3.2), which gives $\Gamma_{\pi}(q ; 0)=i \gamma_{5} E_{\pi}\left(q^{2}\right)$, one can easily show that:

$$
E_{\pi}\left(q^{2}\right)=\frac{16}{3} g^{2} \int \frac{d^{4} k}{(2 \pi)^{4}} \bar{D}_{\mu \mu}(q-k) \frac{E_{\pi}\left(k^{2}\right)}{k^{2} \bar{A}^{2}\left(k^{2}\right)+\bar{B}^{2}\left(k^{2}\right)} .
$$

Comparing with Eq. (3.8), one concludes:

$$
E_{\pi}\left(q^{2}\right) \sim \bar{B}\left(q^{2}\right)
$$

Therefore, if at $\mathscr{O}\left(\delta^{0}\right)$ the DSE gives $\bar{B}\left(q^{2}\right) \neq 0$, there is a massless pseudoscalar quark-antiquark bound state in the spectrum.

Let us now examine the Ward-Takashi identity at his zeroth order. Using in Eq. (3.5) the $\mathscr{O}\left(\delta^{0}\right)$ quark propagator, BSE kernel and quark-gluon vertex $\Gamma_{\mu}(q, p)=\gamma_{\mu} T^{a}$, one obtains

$$
\int \frac{d^{4} k}{(2 \pi)^{4}}\left\{g^{2} \bar{D}_{\mu v}\left(q_{+}-k\right)\left[\gamma_{\mu} T^{a} \bar{S}(k) \gamma_{v} T^{a} \gamma_{5}\right]_{A B}+g^{2} \bar{D}_{\mu v}\left(q_{-}-k\right)\left[\gamma_{5} \gamma_{\mu} T^{a} \bar{S}(k) \gamma_{v} T^{a}\right]_{A B}\right\}
$$




$$
\begin{aligned}
& =-\int \frac{d^{4} k}{(2 \pi)^{4}} g^{2}\left(\gamma_{\mu} T^{a}\right)_{A C} \bar{D}_{\mu v}(q-k)\left(\gamma_{v} T^{a}\right)_{D B}\left[\bar{S}\left(k_{+}\right) \gamma_{5}+\gamma_{5} \bar{S}\left(k_{-}\right)\right]_{C D} \\
& =\int \frac{d^{4} k}{(2 \pi)^{4}} g^{2} \bar{D}_{\mu v}(q-k)\left\{\left[\gamma_{\mu} T^{a} \bar{S}\left(k_{+}\right) \gamma_{v} T^{a} \gamma_{5}\right]_{A B}+\left[\gamma_{5} \gamma_{\mu} T^{a} \bar{S}\left(k_{-}\right) \gamma_{v} T^{a}\right]_{A B}\right\} .
\end{aligned}
$$

Trivial changes of variables establish the identity.

Given that we have a stating point that preserves Goldstone's theorem and satisfies the axialvector Ward-Takahashi identity, let us examine the application of the cutting scheme of Ref. [3] to the next order in DPT. Application of the scheme to the $\mathscr{O}\left(\delta^{4}\right)$ quark self-energy amounts to cut sequentially the dressed internal quark lines in the two lower graphs of Fig. 3. This process leads to the following $\mathscr{O}\left(\delta^{4}\right)$ quark-antiquark scattering kernel:

$$
K_{4}(q, k ; P)=K_{4}^{A}(q, k ; P)+K_{4}^{n A}(q, k ; P),
$$

where $K_{4}^{A}(q, k ; P)$ and $K_{4}^{n A}(q, k ; P)$ correspond respectively to the Abelian $\Sigma_{4}^{A}(q)$ and non-Abelian $\Sigma_{4}^{n A}(q)$ contributions to the quark self-energy; they can be written as:

$$
\begin{gathered}
{\left[K_{4}^{A}(q, k ; P)\right]_{A C, D B}=g^{2} \bar{D}_{\mu v}(q-k)\left\{\left[\gamma_{\mu} T^{a}\right]_{A C}\left[\Gamma_{v}^{a A}\left(k_{-}, q_{-}\right)\right]_{D B}+\left[\Gamma_{\mu}^{a A}\left(k_{+}, q_{+}\right)\right]_{A C}\left[\gamma_{v} T^{a}\right]_{D B}\right\}} \\
-g^{4} \int \frac{d^{4} l}{(2 \pi)^{4}} \bar{D}_{\mu v}(l) \bar{D}_{\rho \sigma}(q-p+l)\left[\gamma_{\mu} T^{a} S\left(k_{+}-l\right) \gamma_{\rho} T^{b}\right]_{A C}\left[\gamma_{v} T^{b} S\left(q_{-}+l\right) \gamma_{\sigma} T^{a}\right]_{D B},(3.14)
\end{gathered}
$$

where in the last equation we used the explicit expression for $\Gamma^{a A}(k, q)$ given in Eq. (2.25), and

$$
\left[K_{4}^{n A}(q, k ; P)\right]_{A C, D B}=g^{2} \bar{D}_{\mu v}(q-k)\left\{\left[\gamma_{\mu} T^{a}\right]_{A C}\left[\Gamma_{v}^{a n A}\left(k_{-}, q_{-}\right)\right]_{D B}+\left[\Gamma_{\mu}^{a n A}\left(k_{+}, q_{+}\right)\right]_{A C}\left[\gamma_{v} T^{a}\right]_{D B}\right\} .
$$

Replacing these into Eq. (3.5) and using $\Gamma^{a}(k, q)=\Gamma^{a A}(k, q)+\Gamma^{a A}(k, q)$, after anticommuting $\gamma_{5}$ with several $\gamma$ matrices and trivial changes of variables, the Ward-Takahashi identity can be established at this order as well.

In principle, there are dressed quark lines running in the dressed gluon propagator $\bar{D}_{\mu \nu}$ and cutting them gives rise to annihilation processes which contribute to isoscalar channels. These are particularly crucial ingredients in studies where the non-Abelian axial anomaly plays dominant role, like in the $\eta-\eta^{\prime}$ mass splitting [3, 21]. For flavor non-diagonal meson states we consider here, these annihilation processes do not contribute.

The same scheme just described can be applied to other Ward-Takahashi identies, e.g. those arising from global U(1) symmetries. A particular important example is conservation of the electromagnetic current. The corresponding Ward-Takahashi identity imposes constraints to the electronphoton vertex function, which is a ingredient in the calculation of hadron form factors and quark distribution functions.

Note that the expressions for the kernel at $\mathscr{O}\left(\delta^{4}\right)$ in Eqs. (3.14) and (3.15) are precisely those one would obtain using Feynman rules derived from $\overline{\mathscr{S}}_{I}$, Eq. (2.11). The "extraneous" term $\delta^{2} \bar{\psi} \bar{\Sigma} \psi$, which is subtracted from the original QCD Lagrangian, does not contribute to the kernel: its iteration, $\delta^{4}(\bar{\psi} \bar{\Sigma} \psi)(\bar{\psi} \bar{\Sigma} \psi)$, certainly contributes to $K_{4}$ but, in view of Eq. (2.16), this contribution is cancelled by a same-order term which comes from the mixing of $\delta^{2} \bar{\psi} \bar{\psi} \psi$ with the dressed one-gluon exchange, $\delta^{4}(\bar{\psi} \bar{\Sigma} \bar{\psi})\left(\bar{\psi} \gamma \cdot A^{a} T^{a} \psi\right)\left(\bar{\psi} \gamma \cdot A^{b} T^{b} \psi\right)$. 
Contributions of higher orders in $\delta$ for the DSEs of quark and gluon self-energies and BSE kernels can be obtained using Feynman rules derived from $\overline{\mathscr{S}}_{I}$. They consist of multiple quark and gluon loops, in which cancellations of the terms $\bar{\psi} \bar{\Sigma} \psi$ and $A \bar{\Pi} A$, of the kind described above, will happen at each order. However, one does not need to worry about such cancellation to construct the BSE kernels if one uses the cutting scheme of Ref. [3], which can be implemented in a automated and analytical way. As explained in Ref. [5], one can use the general relation between the BSE kernel and the quark self-energy:

$$
\left[K_{M}\left(x^{\prime}, y^{\prime} ; x, y\right)\right]_{A C, D B}=-\frac{\delta \Sigma_{A B}(x, y)}{\delta S_{C D}\left(x^{\prime}, y^{\prime}\right)},
$$

where $\Sigma(x, y)$ is obtained from an effective action in the presence of a bilocal source for $\bar{\psi}(x) \psi(y)$ so that translational invariance is recovered only after removing the source [22]. The reason for stating the expression in coordinate space is to make clear that one has the correct number of independent space-time coordinates to produce, under Fourier transformation, a $K_{M}(q, k ; P)$ with the correct total momentum $P$ dependence.

\section{Conclusions and Perspectives}

In this communication we have introduced DPT: a perturbative approach based on the idea of using dressed propagators, instead of free propagators like in ordinary perturbation theory. As we have shown, at lowest order $\mathscr{O}\left(\delta^{0}\right)$, DPT is very similar to the rainbow-ladder truncation scheme, at $\mathscr{O}\left(\delta^{4}\right)$, the next contributing order, vertex corrections are introduced. Higher order corrections can be calculated in systematic and controllable way, very much similar to ordinary perturbation theory. We have shown explicitly that Ward-Takahashi identity is obeyed at $\mathscr{O}\left(\delta^{4}\right)$, and Golstone's theorem is respected.

We have not discussed local gauge invariance. This requires the introduction of ghost fields. However, from the discussions presented, it should be clear that DPT is applicable to the DSEs involving ghost fields. Imposing that $\mathscr{O}\left(\delta^{2}\right)$ contributions vanish, like in Eq. (2.16) for the quark and gluon self-energies, the zeroth-order ghost self-energies become determined: they satisfy a self-consistent Dyson-Schwinger equation with bare vertices. But now, Slavnov-Taylor identities must be considered, which replace the Ward-Takahashi identities of local symmetries; they are much more complicated as they involve also aghast propagators, not only quark and gluon propagators. In this respect, the Pinch Technique (or the background field method) can be of great help, as the only Ward-Takahashi appear [23]. Also, we have not considered renormalization issues, because of lack of space. Up to $\mathscr{O}\left(\delta^{4}\right)$, one can show explicitly that DPT is multiplicatively renormalizable [24].

\section{Acknowledgments:}

I would like to thank my students Marco Brito and Fernando Serna for their work in the development of DPT. Work partially financed by Fundação de Amparo à Pesquisa do Estado de São Paulo - FAPESP, Grant Nos. 2009/50180-0 and 2013/01907-0, and Conselho Nacional de Desenvolvimento Científico e Tecnológico - CNPq, Grant No. 305894/2009-9 . 


\section{References}

[1] A. Bashir, L. Chang, I. C. Cloët, B. El-Bennich, Y.-X. Liu, C. D. Roberts and P. C. Tandy, Commun. Theor. Phys. 58, 79 (2012).

[2] R. Alkofer and L. von Smekal, Phys. Rep. 353, 281 (2001).

[3] A. Bender, C. D. Roberts and L. Von Smekal, Phys. Lett. B 380, 7 (1996).

[4] A. I. Davydychev, P. Osland and L. Saks, Phys. Rev. D 63, 014022 (2001).

[5] P. Watson, W. Cassing and P. C. Tandy, Few Body Syst. 35, 129 (2004).

[6] M. S. Bhagwat, A. Höll, A. Krassnigg, C. D. Roberts and P. C. Tandy, Phys. Rev. C 70, 035205 (2004).

[7] H. H. Matevosyan, A. W. Thomas and P. C. Tandy, Phys. Rev. C 75, 045201 (2007).

[8] R. Alkofer, C. S. Fischer, F. J. Llanes-Estrada and K. Schwenzer, Ann. Phys. 324, 106 (2009)

[9] C. S. Fischer and R. Williams, Phys. Rev. Lett. 103, 122001 (2009).

[10] A. C. Aguilar and J. Papavassiliou, Phys. Rev. D 83, 014013 (2011).

[11] A. Bashir, A. Raya and S. Sanchez-Madrigal, Phys. Rev. D 84, 036013 (2011).

[12] A. Okopinska, Phys. Rev. D 35, 1835 (1987); M. Moshe and A. Duncan, Phys. Lett. B 215, 352 (1988); C. M. Bender and A. Rehban, Phys. Rev. D 41, 3269 (1990); C. M. Bender and K. A. Milton, Phys. Rev. D 38, 1310 (1990); S. K. Gandhi and M. B. Pinto, Phys. Rev. D 49, 4528 (1994); S. Chiku and T. Hatsuda, Phys. Rev. D 58, 076001 (1998), E. Braaten and E. Radescu, Phys. Rev. Lett. 89, 271602 (2002); J. -L. Kneur, M. B. Pinto and R. O. Ramos, Phys. Rev. A 68, 043615 (2003); R. L. S. Farias, G. Krein and R. O. Ramos, Phys. Rev. D 78, 065046 (2008).

[13] G. Krein, D. P. Menezes and M. B. Pinto, Phys. Lett. B 370, 5 (1996); G. Krein, R. S. M. de Carvalho, D. P. Menezes, M. Nielsen and M. B. Pinto, Eur. Phys. J. A 1, 45 (1998).

[14] N. C. Cassol-Seewald, R. L. S. Farias, G. Krein, R. S. Marques de Carvalho, Int. J. Mod. Phys. C 23, 1240016 (2012).

[15] F. Karsch, A. Patkós and P. Petreczky, Phys. Lett. B 401, 69 (1997); J. O. Andersen, E. Braaten and M. Strickland, Phys. Rev. D 63, 105008 (2001).

[16] P. Maris and P. C. Tandy, Nucl. Phys. B (Proc. Suppl.) 161, 136 (2006).

[17] J. Haidenbauer, G. Krein, U. G. Meissner and A. Sibirtsev, Eur. Phys. J. A 33, 107 (2007); Eur. Phys. J. A 37, 55 (2008); J. Haidenbauer, G. Krein, U.-G. Meissner and L. Tolos, Eur. Phys. J A 47, 18 (2011).

[18] G. Krein, A. W. Thomas and K. Tsushima, Phys. Lett. B 697, 136 (2011); K. Tsushima, D. H. Lu, G. Krein and A. W. Thomas, Phys. Rev. C 83, 065208 (2011)

[19] B. El-Bennich, G. Krein, L. Chang, C. D. Roberts and D. J. Wilson, Phys. Rev. D 85, 031502 (2012).

[20] F. Serna, Dressed Perturbation Theory for the Quark Propagator, M.Sc Dissertation, IFT-UNESP, May 2013.

[21] M. S. Bhagwat, L. Chang, Y.-X. Liu, C. D. Roberts, and P. C. Tandy, Phys. Rev. C 76, 045203 (2007).

[22] H. J. Munczek, Phys. Rev. D 52, 4736 (1995).

[23] J. M. Cornwall, J. Papavassiliou and D. Binosi, The Pich Technique and its Applications to Non-Abelian Gauge Theories (Cambridge, 2011).

[24] M. A. Brito, G. Krein and F. Serna, in preparation. 